\title{
Performance analysis of ATM transmission over a DS-CDMA channel
}

T. Timotijevic, J. A. Schormans

Queen Mary and Westfield College

University of London

Dept. of Electronic Engineering, Mile End Road, London E1 4NS, United Kingdom e-mail: (T.Timotijevic, J.A.Schormans)@qmw.ac.uk Tel: +441714153756 Fax: +441819810259

\begin{abstract}
This paper presents numerical results for ATM cell transmission over a DS-CDMA satellite link obtained by mathematical analysis. We show that an increase in capacity can be achieved by using discontinuous transmission detection (DTX) for all ON-OFF sources, thus exploiting the burstiness of ATM traffic. Three scenarios are analysed depending on whether the code is allocated to a user or to a single virtual connection (VC), and whether the transmission rate for different connections is the same or varies across the system. System performance is expressed in terms of link efficiency and relative capacity increase. Initial results show how different transmission rates and loads affect the system performance.
\end{abstract}

Keywords

ATM, DS-CDMA capacity, traffic analysis, ATM over satellite 


\section{INTRODUCTION}

We describe three architectural scenarios that exploit the bursty nature of ATM traffic to increase the broadband DS-CDMA satellite system capacity, and analyse their cell-level performance. A multirate wireless DS-CDMA system can accommodate connections of varying data rates by adjusting the different data rates to an intermediate transmission rate $R$ prior to spreading by a pseudo-random or other code of a much higher rate (chip rate). We focus on the system performance of the uplink, from the ATM layer viewpoint, on a satellite link. The results show that the performance on the ATM layer influences the system design on the lower, physical, layer. The bursty nature of ATM is in this case beneficial to the system capacity. The performance is assessed with respect to the system capacity and link efficiency (utilisation). Our aim is to find an optimum scenario with its system design parameters for a given satellite transponder bandwidth.

As CDMA is interference-limited scheme, it can approach and even outperform TDMA in terms of system capacity under conditions that reduce the interference in the system (Elhakeem 1994, Van Nee 1995, Monsen 1995). The capacity increase in a CDMA achievable by DTX has been mainly researched in voice systems (Yang 1994) or multimedia systems with DTX applied only to voice (Fong 1996). Work within RACE 2020 CODIT project, as reported in (Baier 1993, Andermo 1994), applied DTX to other types of traffic. However, no detailed celllevel performance analysis has been presented.

In this study, we investigate the impact of traffic characteristics, as well as the choice of system parameters, on the performance of different scenarios. This serves as a starting point for the development of connection-level control schemes in the satellite IBC networks that use ATM over DS-CDMA. Our mathematical analysis has been validated using a cell level network simulator.

\section{SYSTEM DESCRIPTION}

We assume DS-CDMA uplink, ideal power control and pure ATM (no framing). Only degradation effects intrinsic to CDMA are taken into account, i.e. CDMA multiple-access interference. Our three configuration scenarios are based on:

- code allocation policy: code per user, and code per virtual channel connection (code per VCC);

- transmission rate $R$ across the system: whether it is constant, or varies among users.

In Code per User allocation policy, VCs are multiplexed prior to spectrum spreading and transmission. In Code per VCC, each VCC gets its own spreading code and multiplexing is done "in the air", in the code domain.

Use of the DTX for CDMA system capacity increase relies on the random nature of sources. In our case, statistical multiplexing is achieved by switching off the 
carrier when there the source is idle, resulting in the reduction of the overall system self-interference. The drawback is the need for code and carrier re-synchronisation when the source reactivates. In existing CDMA systems transmission during silent periods is continued at reduced power and bit rate in order to preserve carrier synchronisation. The possible increase in capacity in this case may be lower than when the transmission is completely switched off. This trade-off between capacity and efficiency will be considered in future work.

With DTX employed, the empty cells will still be inserted during active periods of the source due to the difference in the transmission and source data rates. For pure ATM, the link efficiency is measured as the proportion of useful cells. Link efficiency is increased when the number of transmitted empty cells is decreased.

\subsection{Impact of processing gain on system architecture}

The ratio of transmission and chip rates is the CDMA processing gain. It determines the amount of multiple-access interference perceived by a receiver. The approximate formula that binds the processing gain $G_{p}$, signal-to-noise-plusinterference ratio (SNR), maximum number of users that could be accepted in a system while maintaining the specified $S N R\left(C_{\max }\right)$ and $E b / N o$ is (Pursley 1977):

$$
S N R=\left(\frac{C_{\max }-1}{3 G_{p}}+\frac{N_{0}}{2 E_{b}}\right)^{-1} .
$$

The first term above effectively defines multiple-access interference from other users. The quality of service of a connection is related to the $S N R$, and is expressed in terms of the cell loss ratio (CLR). In our model transmitted Eb/No is constant. Depending on whether the transmission rates for different traffic types vary or not, and whether the system allows different QoS for different traffic types, the processing gain $G_{p}$ can be constant or variable.

\subsection{Scenarios}

\subsubsection{Code per User allocation policy, $R=$ const. (Figure 1)}

Multiplexing of VCCs is at the user terminal. Link BER requirement will dictate a limit for CLR at the buffer, which may lead to increase of the queueing delay. The link has to maintain the QoS for the most stringent traffic type. This yields $G_{p}=$ const. across the system. As $R=$ const. all channels have equal bandwidth

\subsubsection{Code per VCC allocation policy, $R=$ const. (Figure 2)}

Each connection gets its own spreading code, hence multiplexing at the terminal is avoided. We can expect only very little or no buffering delay. Different connection types can be transmitted in the same channel due to $R=$ const. QoS cannot vary since all processing gains within a channel must be equal. 


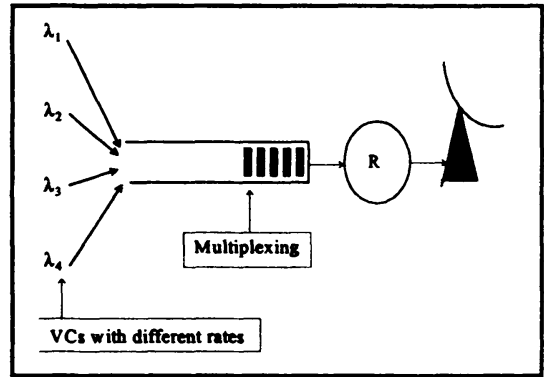

Figure 1 Code per User scenario

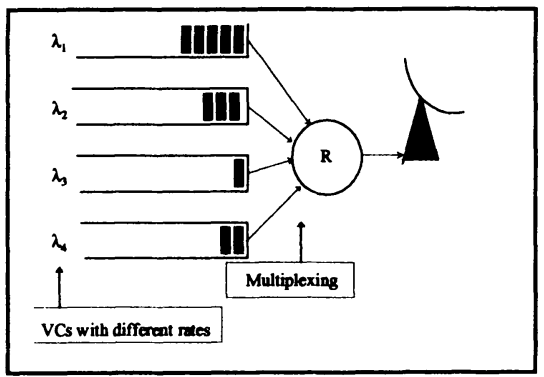

Figure 2 Code per VCC $(R=$ const $)$

\subsubsection{Code per VCC allocation policy, $R<>$ const.}

Each connection has its own spreading code, and different traffic types have different transmission rates. The problem of choosing the optimum $R$ becomes a problem of system channel dimensioning, i.e. determining channel bandwidths for different traffic types and the number of such channels for envisaged corresponding load. Variation of QoS can be achieved by choosing appropriate values of $G_{p}$. Within each channel, the increase of capacity is measured as number of connections of that particular type. Thus capacity increase depends on the maximum number of users $C_{\max , k}$, QoS for type $k$, and its activity factor $a_{k}$. Illustration of this scenario differs from Figure 2 only in that $R<>$ const.

\subsection{DTX}

The DTX mechanism turns the transmission off after a hangover period if the queue in the buffer is empty. Hangover is equal to the cell inter-arrival time (IAT) of the slowest connection (in case of the Code per User scenario), or just the cell inter-arrival time of the corresponding connection (in Code per VCC scenarios). Signal transmission time is also extended by code re-synchronisation. The effect of acquisition and hangover on busv and idle periods is illustrated on Figure 3.

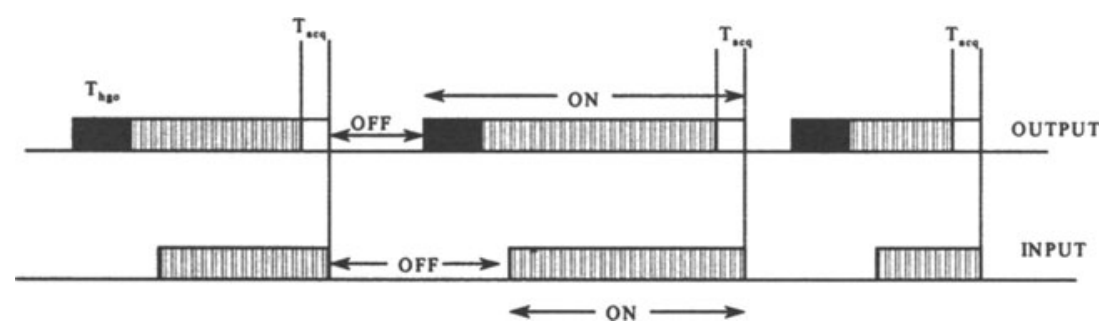

Figure 3 Busy and idle periods of an input stream and a multiplexer output.

\section{ANALYSIS}

The analysis is performed on the cell level in discrete time. Sources are modelled as ON-OFF streams with deterministic interarrival times and geometrically distributed state sojourn times. The multiplexer is modelled as an infinite queue with fixed service time that determines the slot rate at its output and geometrically 
distributed state sojourn times. Each connection type has different probabilities of $\mathrm{ON}$ and OFF periods, with different cell rates in $\mathrm{ON}$ states. Variables used in derivations of link efficiency (subscript $k$ used in Scenarios 2 and 3 analysis) are:

$K$

$\lambda_{k}$

$S R, S R_{k}$ $P C R, P C R_{k}$

$T_{B} T_{P}$

$T_{\text {hgo, }} T_{\text {hgo.k }}$

$T_{\text {aca }}$

$p_{\text {ON.K }} p_{\text {OFF, }}$

$T_{\text {ON,k }} T_{\text {OFF, }}$

$a_{k}$

$N, N_{k} N_{k}(i)$

$C_{\max }, C_{\max . k}$

$m_{k}$

$M$

$W, W_{k}$ number of traffic types (for numerical analysis $K=3$ )

mean arrival rate of the $k$ th connection

slot rate expressed in [cells/s]

peak cell rate of a source during busy $(O N)$ period

mean duration of a busy period of a multiplexer in Scenario 1

mean hangover period

mean acquisition period

probability that the connection $k$ is ON/OFF,

mean ON and OFF periods of the $k$ th connection,

activity factor of connection (traffic) type $k$

number of connections (of traffic type $k$ in Scenario 2;

connections of traffic type $k$ in $i$ th channel in Scenario 3)

maximum theoretical number of signals in a DS-CDMA channel number of channels for carrying traffic type $k$ total number of channels

total bandwidth, bandwidth of channel carrying type $k$

\subsection{Code per User scenario}

We are interested in efficiency of one user (link) and some equivalent 'aggregate' efficiency of the whole channel. Since in this scenario all users and channels are identical, efficiency of the channel will be the same for all users, and equal to the efficiency of the system. Efficiency with and without DTX (continuous transmission) is:

$$
\begin{aligned}
& E f f_{D T X}=\frac{\left(T_{B}+T_{I}\right) \cdot \sum_{k=1}^{K} \lambda_{k}}{S R \cdot\left(T_{B}+T_{h g o}+T_{a c q}\right)} . \\
& \text { Eff }_{C O N T}=\frac{\sum_{k=1}^{K} \lambda_{k}}{S R} \equiv \rho .
\end{aligned}
$$

It is assumed that all users have the same mean busy and idle periods at the output of the multiplexer, although in reality it may be possible that the connections of a user will not all be active at all times.

\subsubsection{Available capacity and CAC}

Available unused capacity can be expressed as: 


$$
\frac{\Delta C}{C_{\max }}=1-\frac{U(\rho)}{C_{\max }} \cdot \frac{T_{B}+T_{a c q}+T_{h g o}}{T_{B}+T_{I}} .
$$

$U(\rho)$ is a maximum number of users required to achieve the total load on the satellite of $\rho$. Depending on activity factors $a_{k}$ of traffic types $k$, available capacity can be fully utilised by statistical multiplexing of extra users in the code domain. Assuming the number of users (producing load $\rho$ ) of type $k$ is $U_{k}(\rho)$, then the number of extra users of traffic type $j$ that can be accepted above the theoretical value $C_{\text {max }}, \Delta U_{\text {Jmax }}(\rho)$ is:

$$
\Delta U_{j, \max }=\frac{C_{\max }-\sum_{k} a_{k} \cdot U_{k}(\rho)}{a_{j}} .
$$

Expression (5) treats a general case. In Code per User scenario, some "equivalent" activity has to be found before the capacity increase can be assessed. To do this, it is necessary to determine the number of users $U(\rho)$ that produces the load $\rho$, which is easily done in queueing theory. Expression (5) forms the basis for a connection admission control (CAC) in a satellite IBC system. The controller can assess the available capacity using the known activity factors, which can be measured on-line or provided as traffic descriptors. When a new connection requests access, the CAC algorithm accepts it if $\Delta U_{j, \text { max }}(\rho)>1$.

\subsection{Code per VCC, $R$ uniform across the system}

In this scenario the server mean idle and busy periods differ from source $\mathrm{ON}$ and OFF periods by the hangover and mean code acquisition time. The cells are delayed by the mean acquisition time, which is the same across the system. Efficiency with and without DTX are given by Equations 6 and 7:

$$
\begin{gathered}
E f_{D T X}=\left[1+\frac{\sum_{k=1}^{K} N_{k}\left(T_{a c q}+T_{h g o, k}\right) \cdot S R \frac{\Delta t}{T_{O N, k}+T_{O F F, k}}+\sum_{k=1}^{K} N_{k}\left(S R-P C R_{k}\right) p_{O N, k} \cdot \Delta t}{\sum_{k=1}^{K} N_{k} \lambda_{k} \Delta t}\right]^{-1} \\
=\left\{1+\frac{\sum_{k=1}^{K} N_{k} \cdot p_{O N, k} \cdot\left[S R \cdot\left(1+\frac{T_{a c q}+T_{h g o, k}}{T_{O N, k}}\right)-P C R_{k}\right]}{\sum_{k=1}^{K} N_{k} \lambda_{k}}\right]^{-1}
\end{gathered}
$$


$E f f_{C O N T}=\frac{\sum_{k=1}^{K} N_{k} \cdot \lambda_{k}}{C_{\max } \cdot S R}$

\subsection{Code per VCC, $R$ varies across the system}

For each connection type $k$ of total $K$ types, there are $m_{k}$ channels of corresponding bandwidth $W_{k}$. Channel bandwidths are determined by the QoS requirements of different connection types and their transmission rates. Each channel can have a theoretical maximum of $C_{\text {max. }}$ connections, and a number of active users in the channel is $N_{k}(i), i=1, \ldots, m_{k}$. The acquisition time of a code varies according to the QoS requirements and transmission rate (since it depends on the probability of false alarm and probability of detection (Holmes, 1982)). Therefore, each connection type $k$ has a different code mean acquisition time $T_{\text {ocq.. }}$. We have:

$E f_{D T X}=\left[1+\frac{\sum_{k=1 i=1}^{K} \sum_{k}^{m_{k}} N_{k}(i)\left(T_{a c q}+T_{h g q k}\right) \cdot S R_{k} \cdot \frac{\Delta}{T_{O N, k}+T_{O F F, k}}+\sum_{k=1 i=1}^{K m_{k}} N_{k}(i)\left(S R_{k}-P C R\right) p_{O N, k} \cdot \Delta t}{\sum_{k=1 i=1}^{K \sum_{k}} N_{k}(i) \lambda_{k}(i)}\right]^{-1}$

$=\left[1+\frac{\sum_{k=1}^{K} \sum_{i=1}^{m_{k}} N_{k}(i) p_{O N, k}\left[S R_{k} \cdot\left(1+\frac{T_{a c q, k}+T_{h g o, k}}{T_{O N, k}}\right)-P C R_{k}\right]}{\sum_{k=1}^{K} \sum_{i=1}^{m_{k}} N_{k}(i) \lambda_{k}}\right]^{-1}$.

$E_{C O N T}=\frac{\sum_{k=1}^{K} \sum_{i=1}^{m_{k}} N_{k}(i) \cdot \lambda_{k}}{\sum_{k=1}^{K} m_{k} \cdot C_{\max , k} \cdot S R_{k}}$

\section{RESULTS}

To obtain numerical results we used approximations of $K=3$ types of traffic: voice, video and data, with the parameters:

- voice: $64 \mathrm{~kb} / \mathrm{s}$ source, with $\mathrm{T}(\mathrm{on})=1.54 \mathrm{~s}$ and $\mathrm{T}(\mathrm{off})=2.75 \mathrm{~s}, a_{1}=0.36$;

- video: $384 \mathrm{~kb} / \mathrm{s}$ source with $\mathrm{T}(\mathrm{on})=20 \mathrm{~s}$ and $\mathrm{T}$ (off) $=6.5 \mathrm{~s}, a_{2}=0.75$;

- data: $4 \mathrm{Mb} / \mathrm{s}$ source with $\mathrm{T}(\mathrm{on})=1.79 \mathrm{~ms}$ and $\mathrm{T}(\mathrm{off})=26.7 \mathrm{~s}, a_{3}=0.09$. 
For any traffic source type, when in the ON state, cells are generated at a constant rate; no cells are generated in the OFF state. A number of different transmission rates are being considered: $64 \mathrm{~kb} / \mathrm{s}, 384 \mathrm{~kb} / \mathrm{s}, 512 \mathrm{~Kb} / \mathrm{s}, 1 \mathrm{Mb} / \mathrm{s}, 1,5 \mathrm{Mb} / \mathrm{s}, 1,8 \mathrm{Mb} / \mathrm{s}$, $2 \mathrm{Mb} / \mathrm{s}$ and $4 \mathrm{Mb} / \mathrm{s}$. We assumed $S N R=4.5 \mathrm{~dB}$ and $E b / N o=12 \mathrm{~dB}$ for all types. At the moment only results for the first two scenarios are available. For them, $64 \mathrm{~kb} / \mathrm{s}$ rate is below the mean rates of video and data traffic, causing buffer overflows. Since the loads on different connections depend on the mean arrival and slot rates, and the mean arrival rates in our model are fixed, then for higher transmission (slot) rates only low loads can be achieved. This is because the maximum number of users or connections $C_{\max }$ is fixed.

\subsection{Efficiency}

Numerical results obtained by analysis are plotted on Figures 4-7. In all graphs, efficiency without DTX is essentially the load of the system (ratio of cell arrival and cell service rates).

\subsubsection{Code per User scenario}

In the Code per User scenario efficiency of the system with DTX decreases with the increase of the transmission rate (from 0.82 at $512 \mathrm{~Kb} / \mathrm{s}$ and $70 \%$ load to 0.10 at $4 \mathrm{Mb} / \mathrm{s}$ and $10 \%$ load, see Figure 4 ). The efficiency improvement is constant (17\%) for all transmission rates greater than $384 \mathrm{~Kb} / \mathrm{s}$. Hence for a more efficient system it is necessary that the transmission rate is as low as possible (so that fewer empty cells are transmitted). However, for low transmission rates $(64 \mathrm{~Kb} / \mathrm{s}$ and 384 $\mathrm{Kb} / \mathrm{s}$ on Figure 4 ) the system with DTX is overloaded (efficiency with DTX becomes greater than 1 , which is impossible). This is caused by the generation of additional cell rate due to the overheads, and results in the 'aggregate' load being greater than 1. This means that a traffic mix is important in determining the transmission rate for this scenario, and it has to be low enough to keep the system efficient, while maintaining the aggregate load below 1 .

\subsubsection{Code per VCC, $R=$ const.}

Efficiency of this scenario has much higher values than in Code per User scenario (up to 0.99 for $70 \%$ loads and $384 \mathrm{~Kb} / \mathrm{s}$ transmission rate, Figure 5), for all loads and transmission rates. Moreover, the efficiency improvement is much higher than that in Code per User: values of $40 \%$ are obtained for $70 \%$ loads. The effect of connection activity factor is predictable: for a constant number of connections of a given traffic type, the efficiency improvement will increase with the increase of the number of connections with lower activity factor. However, if the activity factor has very low values, the number of overhead cells transmitted in comparison to the useful cells starts to increase, and the efficiency decreases again. For example, efficiency decreases more quickly if video connections are kept constant and the number of voice connections is increased, than when the voice connections are kept constant and video are increased. This is due to the proportion of overhead present in the connections with lower activity factors: in less active sources, the proportion of DTX overhead is higher (the duration of active states is closer to the 
duration of overhead periods). Hence, the capacity gained through having connections of lower activity factors has its cost in the comparatively lower efficiency (with DTX). Overall, Code per VCC scenario outperforms Code per User scenario with respect to efficiency.

\subsection{Capacity}

The relative capacity increase in Code per VCC scenarios is measured as number of connections of one particular type. We compare the values of relative capacity increase for different connection types. For the same loads, transmission rates and channel bandwidths, Code per VCC outperforms the Code per User scenario in terms of voice and data connections (Figures 8-11). However, in terms of video users, the relative capacity increase in Code per VCC scenario is comparable to or lower than the capacity increase in Code per User scenario. This is due to the fact that the activity factor of video sources $(0.75)$ is close to the activity factor of multiplexed connections in Code per User scenario (0.86), while the available capacity for a particular traffic load is higher in the Code per User than in Code per VCC scenario, because fewer users are necessary in Code per User scenario to achieve the same loads.

\subsubsection{Code per User}

The capacity improvement decreases with the load and transmission rate. Total system capacity is highest for the $384 \mathrm{~Kb} / \mathrm{s}$ transmission rate and the $25 \mathrm{MHz}$ channel bandwidth.

\subsubsection{Code per VCC}

Capacity increase is lower at higher transmission rates for the same loads and channel bandwidths (Figures 9-10). As expected, for a given channel bandwidth and transmission rate, the highest capacity increase results from the connections of lowest value of activity factor (data traffic), and all curves drop with the traffic load. This is because higher loads have less unused capacity available for further statistical multiplexing of the connections.

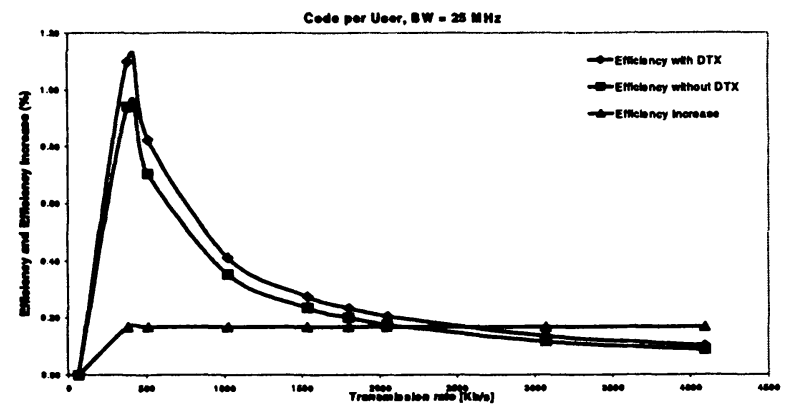

Figure 4. 


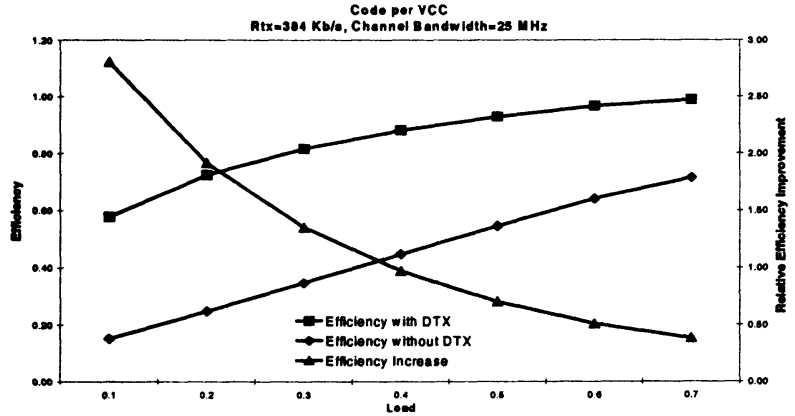

Figure 5

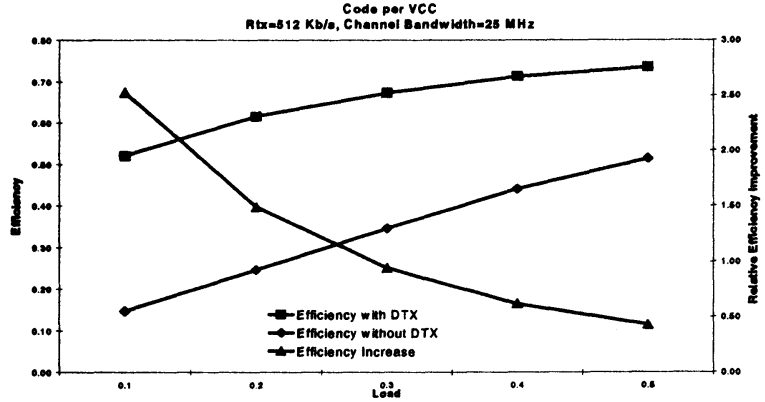

Figure 6

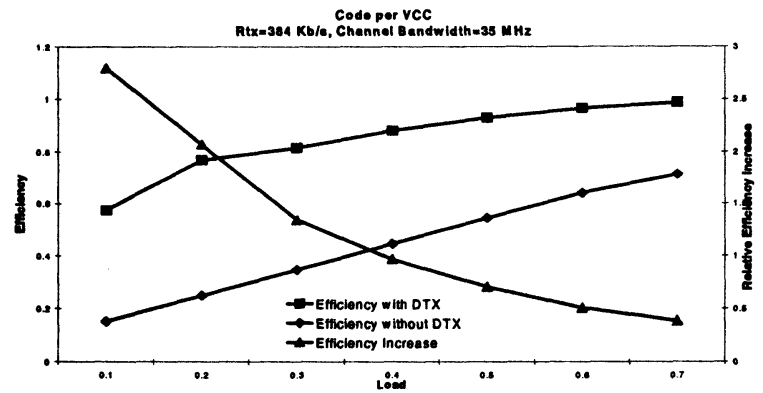

Figure 7.

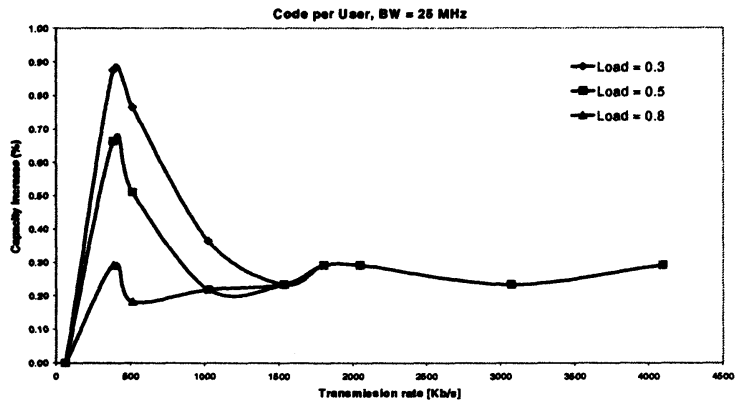

Figure 8. 


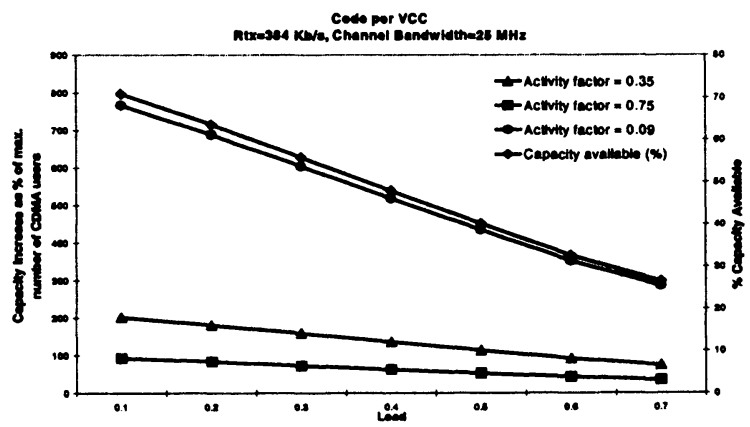

Figure 9.

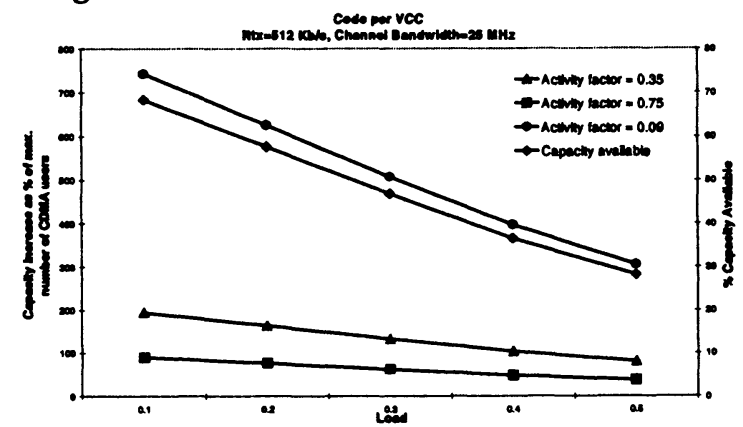

Figure 10.

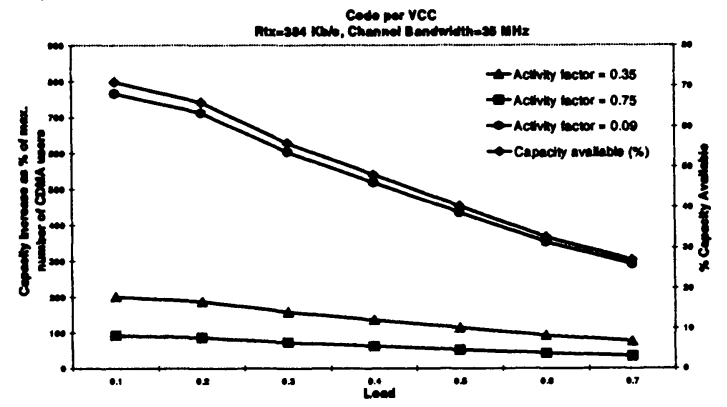

Figure 11.

\section{SUMMARY AND CONCLUSIONS}

Three configuration scenarios are described that use DTX and exploit bursty nature of ATM broadband traffic to increase the CDMA system capacity. We presented numerical results that establish performance gains for two of the three described scenarios. The results show how the system relative capacity increase changes with traffic load, transmission rates and the traffic activity factor, and how efficiency changes with load and transmission rate. The idea for a simple CAC algorithm in a CDMA broadband system that exploits sources' activity factor has been presented. 
Preliminary conclusion is that Code per VCC scenario outperforms Code per User scenario. Choice of transmission rate directly affects the system capacity and efficiency, but it also determines the buffering delay in the multiplexer, crucial for the delay-sensitive traffic particularly in satellite systems. The future work will investigate imposed delays and the third scenario - Code per VCC when $R$ varies across the system - and will aim to identify the rules for the choice of the system parameters based on the expected traffic mix, traffic types, and the optimal scenario.

\section{REFERENCES}

Andermo, P.G. and Brismark, G. (1994). CODIT, a testbed project evaluating DSCDMA for UMTS/FPLMTS. Proc. of the IEEE $44^{\text {th }}$ Vehicular Technology Conference "Creating tomorrow's mobile systems", 8-10 June 1994, Stockholm, Sweden, Ch. 389, 21-5

Baier, A. and Panzer, H. (1993). Multi-rate DS-CDMA radio interference for thirdgeneration cellular systems. Proc. of the $7^{\text {th }}$ IEE European Conference on Mobile and Personal Communications, 13-15 December 1993, Brighton, England, Ch. 46, Conf. Pub. Np. 387, 255-9

Elhakeem, A.K., Di Girolamo, R., Bdira, I.B. and Talla, M. (1994). Delay and throughput characteristics of TH, CDMA, TDMA and hybrid networks for multipath faded data transmission channels. IEEE JSAC Vol. 12/4, 622-37

Fong, M.H., Bhargava, V.K. and Wang, Q. (1996). Concatenated orthogonal/PN spreading sequences and their application to cellular DS-CDMA systems. IEEE JSAC, Vol. 14/3, 547-58

Holmes, J. K. (1982). Coherent Spread Spectrum Systems. John Wiley \& Sons Inc.

Monsen, P. (1995). Multiple-access capacity in mobile user satellite systems. IEEE JSAC, Vol. 13/2, 222-31

Pursley, M.B. (1977). Performance analysis for phase-coded spread-spectrum multiple-access communications - Part I: System analysis. IEEE Transactions on Communications, Vol. COM-25, 795-9

Van Nee, R., Van Wolfwinkel, R.N., and Prasad, R. (1995). Slotted ALOHA and code division multiple access techniques for land-mobile satellite personal communications. IEEE JSAC, Vol.13/2, 382-8

Yang, W.B. and Geraniotis, E. (1994). Admission policies for integrated voice and data traffic in CDMA packet radio networks. IEEE JSAC, Vol. 12/4, 654-64 